\title{
Critical Path Analysis of Two-channel Interleaved Digital MASH $\Delta \Sigma$ Modulators
}

\author{
Ameya Bhide and Atila Alvandpour
}

\section{Linköping University Post Print}

\section{Tweet}

N.B.: When citing this work, cite the original article.

(C2015 IEEE. Personal use of this material is permitted. However, permission to reprint/republish this material for advertising or promotional purposes or for creating new collective works for resale or redistribution to servers or lists, or to reuse any copyrighted component of this work in other works must be obtained from the IEEE:

Ameya Bhide and Atila Alvandpour, Critical Path Analysis of Two-channel Interleaved Digital MASH $\Delta \Sigma$ Modulators, 2013, 2013 NORCHI, 11-12 November, 2013, Vilnius, Lithuania, (), , $1-4$.

http://dx.doi.org/10.1109/NORCHIP.2013.6702009

Postprint available at: Linköping University Electronic Press http://urn.kb.se/resolve?urn=urn:nbn:se:liu:diva-120304 


\title{
Critical Path Analysis of Two-channel Interleaved Digital MASH $\Delta \Sigma$ Modulators
}

\author{
Ameya Bhide and Atila Alvandpour \\ Department of Electrical Engineering, Linköping University, \\ SE-58183, Linköping, Sweden, Email: \{ameya,atila\} @isy.liu.se
}

\begin{abstract}
Implementation of wireless wideband transmitters using $\Delta \Sigma$ DACs requires very high speed modulators. Digital MASH $\Delta \Sigma$ modulators are good candidates for speed enhancement using interleaving because they require only adders and can be cascaded. This paper presents an analysis of the integrator critical path of two-channel interleaved $\Delta \Sigma$ modulators. The bottlenecks for a high-speed operation are identified and the performance of different logic styles is compared. Static combinational logic shows the best trade-off and potential for use in such high speed modulators. A prototype 12-bit second order MASH $\Delta \Sigma$ modulator designed in $65 \mathrm{~nm}$ CMOS technology based on this study achieves $9 \mathrm{GHz}$ operation at $1 \mathrm{~V}$ supply.
\end{abstract}

\section{INTRODUCTION}

The use of high speed digital $\Delta \Sigma$ modulators (DDSMs) has been reported in many $\Delta \Sigma$ DAC based transmitters for WLAN, WiFi, UMTS and WiMAX radio standards [1-4]. The speed of these DDSMs has so far been limited to $5.4 \mathrm{GHz}$ [2]. In order to adopt a similar approach for high-data-rate and wideband standards like WiMedia UWB and WiGIG $60-\mathrm{GHz}$ radio, DDSMs that operate at around $10 \mathrm{GHz}$ are required.

MASH DDSMs are very stable and are good candidates for high-speed operation since they are basically accumulators that can be pipelined and cascaded. Nevertheless, a conventional MASH DDSM, shown in Fig. 1, is not suitable for arriving at a $10 \mathrm{GHz}$ operation in a $65 \mathrm{~nm}$ CMOS process. Timeinterleaving of MASH DDSMs is thus required to enhance the speed by making used of relaxed timing constraints and a reduced clock frequency. Only the final multiplexing in the DDSM occurs at the full speed. A $10 \mathrm{GHz}$ throughput can be achieved by using a large number of interleaved channels, however this moves the entire complexity to the multiplexing and multiphase clock generation. When used in a $\Delta \Sigma$ DAC, the DAC dynamic performance is limited by the multiplexing. On the other hand, using only two-channel interleaving (Fig. 2) simplifies the final multiplexing and the clock generation but this pushes the logic to operate at a half-rate clock, which is still a challenging task. A $\Delta \Sigma$ DAC that utilizes this strategy was previously presented in [5]. A study of the delays within the integrator is essential for achieving a high speed using only two-channel interleaving and is the main focus of this paper.

The work presented in [6] primarily addresses only the critical path across the cascaded stages of interleaved MASH DDSMs. The critical path within the integrator of every MASH stage itself was not analyzed as the speed targeted was a moderate $2.5 \mathrm{GHz}$ using a large number of channels (612 channels). This work investigates the speed limitation of

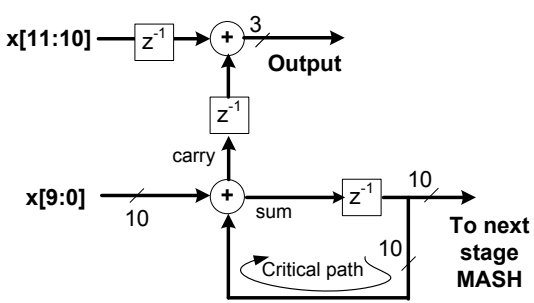

Fig. 1. Conventional MASH $\Delta \Sigma$ modulator with a 12-bit-input 3-bit-output.

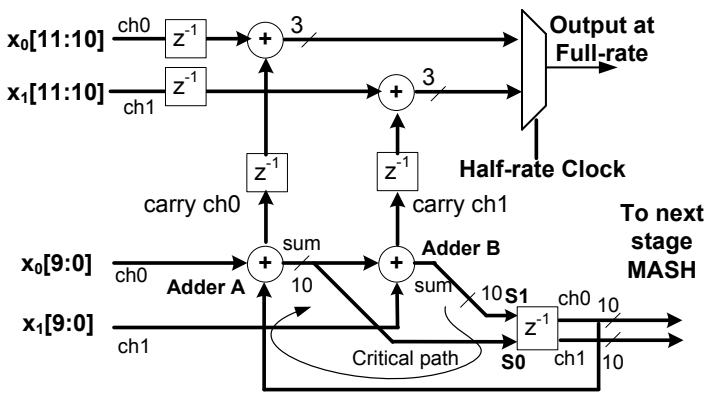

Fig. 2. Equivalent two-channel interleaved MASH $\Delta \Sigma$ modulator working with a half-rate clock.

the two-channel integrator and compares the performance of different logic styles.

\section{Modulator Critical Path}

Consider Fig. 3 that shows one N-bit pipeline stage of the two back-to-back adders $\mathrm{A}(\mathrm{Ch} 0)$ and $\mathrm{B}(\mathrm{Ch} 1)$ in more detail. Both the adders are individually constructed from 1bit adders $A_{0}-A_{N-1}$ and $B_{0}-B_{N-1}$. Outputs $S_{0}$ and $S_{1}$ are the running sum of the integrator for channel 0 and channel 1 respectively. The critical path lies between the flipflops $\mathrm{FF}_{\mathrm{S} 0}$ (start) and $\mathrm{FF}_{\mathrm{SN}-1}$ (end). The carry signals from both the adders move in an upward direction while the sum moves in a lateral direction. From this figure, an important observation about the characteristics of the two adders can be made. Adder $\mathrm{A}$ is required to generate the sum and carry outputs with equal delay while only the carry generation is critical for Adder B like is the case in conventional adders. To understand this better, it can be seen that the worst case delay can result from three different types of paths. In the first case, it results mainly from the carry chain of A, e.g. the path from $\mathrm{FF}_{\mathrm{S} 0} \rightarrow \mathrm{A}_{0} \rightarrow \mathrm{A}_{1} \ldots \rightarrow \mathrm{A}_{\mathrm{N}-1} \rightarrow \mathrm{B}_{\mathrm{N}-1} \rightarrow \mathrm{FF}_{\mathrm{SN}-1}$. In the second case, the delay mainly comes from the carry 


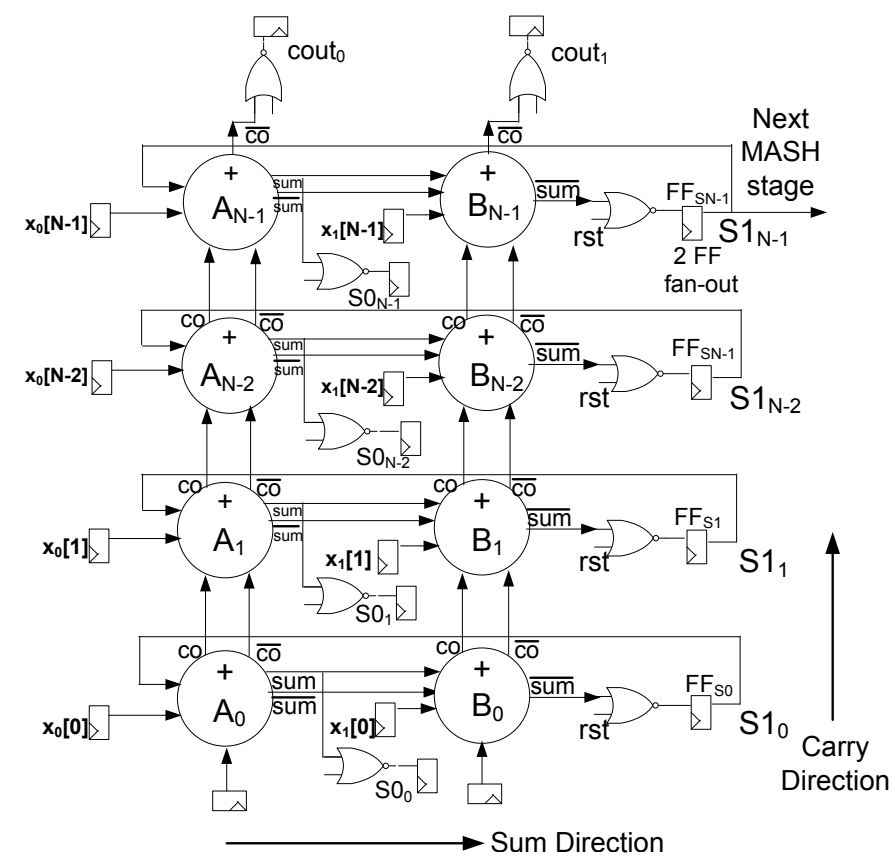

Fig. 3. N-bit deep Integrator Pipeline. Critical path is from at flop $\mathrm{FF}_{\mathrm{S} 0}$ to flop $\mathrm{FF}_{\mathrm{SN}-1}$.

chain of $\mathrm{B}$, e.g. $\mathrm{FF}_{\mathrm{S} 0} \rightarrow \mathrm{A}_{0} \rightarrow \mathrm{B}_{0} \rightarrow \mathrm{B}_{1} \ldots \rightarrow \mathrm{B}_{\mathrm{N}-1} \rightarrow \mathrm{FF}_{\mathrm{SN}-1}$. In the final case, the delay comes partially from the carry chain of $\mathrm{A}$ and partially from the carry chain of $\mathrm{B}$, e.g. $\mathrm{FF}_{\mathrm{S} 0} \rightarrow \mathrm{A}_{0} \rightarrow \mathrm{A}_{1} \rightarrow \mathrm{B}_{1} \ldots \rightarrow \mathrm{B}_{\mathrm{N}-2} \rightarrow \mathrm{B}_{\mathrm{N}-1} \rightarrow \mathrm{FF}_{\mathrm{SN}-1}$. In the second and the third cases, it becomes especially important that the sum generated by the 1-bit adders $\mathrm{A}_{0}-\mathrm{A}_{\mathrm{N}-1}$ be transferred to adder $\mathrm{B}$ as fast as possible. Thus, A has both its outputs, carry and sum in the critical path making it inherently slower than B.

It can be further seen that even within adders A and B, the individual 1-b adders are inherently different. In adder $\mathrm{A}, \mathrm{A}_{0}$ is the slowest cell since it needs to generate the inverted inputs and also the complementary outputs for sum and carry with equal delay. Hence the fan-out of $\mathrm{A}_{0}$ is the highest. Adders $A_{1}-A_{N-2}$ have the same fan-out as $A_{0}$ but faster by one inverter delay as their complementary inputs are already available. $\mathrm{A}_{\mathrm{N}-1}$ is the fastest since it is required to generate only three outputs i.e. $\overline{\mathrm{co}}$, sum, $\overline{\text { sum }}$ and hence has a lesser fan-out. A similar analysis of adder $\mathrm{B}$ shows that $\mathrm{B}_{0}-\mathrm{B}_{\mathrm{N}-2}$ are of the same type and slower than $\mathrm{B}_{\mathrm{N}-1}$. Thus, in a one N-bit deep integrator pipeline, the 1-bit adders can be written in their order of decreasing delays as follows:- $A_{0}>A_{1}$ $\mathrm{A}_{\mathrm{N}-2}>\mathrm{A}_{\mathrm{N}-1}>\mathrm{B}_{0}-\mathrm{B}_{\mathrm{N}-2}>\mathrm{B}_{\mathrm{N}-1}$.

While these observations form the basis for designing the 1-bit adders, a further improvement in delay is possible by noting the fact that the sum outputs of $\mathrm{B}_{0}-\mathrm{B}_{\mathrm{N}-2}$ are not in the critical path. Hence it is possible to reduce the drive strength on these nodes. This improves the delay from sum outputs of $\mathrm{A}_{0}-\mathrm{A}_{\mathrm{N}-2}$ to the carry outputs of $\mathrm{B}_{0}-\mathrm{B}_{\mathrm{N}-2}$ respectively as now there is a reduced capacitance on this path. A similar situation exists with $\overline{c o}$ output of $A_{\mathrm{N}-1}$ and hence this node can be also
TABLE I

MAXIMUM EFFECTIVE ACHIEVED SPEED AS A FUNCTION OF THE PIPELINE DEPTH IN A 2-CHANNEL INTERLEAVED MODULATOR.

\begin{tabular}{|c|c|c|c|c|c|}
\hline $\begin{array}{l}\text { Pipeline } \\
\text { Depth } \\
\text { D }\end{array}$ & $\begin{array}{c}\text { Overhead } \\
\text { Delays }\end{array}$ & $\begin{array}{c}\text { Total } \\
\text { Adder } \\
\text { Delay }(p s) \\
\mathbf{T}_{\text {ad }}\end{array}$ & $\begin{array}{c}\text { Total }^{* *} \\
\text { Path } \\
\text { Delay(ps) } \\
\mathbf{T}_{\mathbf{p}}\end{array}$ & $\begin{array}{c}\text { Effective } \\
\text { Speed } \\
(\mathbf{G H z}) \\
2 / \mathbf{T}_{\mathbf{p}} \\
\end{array}$ & $\begin{array}{c}\text { Avg. 1-b } \\
\text { Adder } \\
\text { Delay(ps) } \\
\mathrm{T}_{\text {ad }} /(\mathbf{D + 1})\end{array}$ \\
\hline 1 & 78 & 110 & 188 & 10.6 & 55 \\
\hline 2 & 88 & 130 & 219 & 9.1 & 44 \\
\hline 3 & 88 & 180 & 268 & 7.4 & 45 \\
\hline 4 & 88 & 200 & 288 & 6.9 & 40 \\
\hline 5 & 88 & 237 & 325 & 6.1 & 39 \\
\hline
\end{tabular}

Overhead Delays include-

a) Flop delay $=30$ ps for $\mathrm{D}=1 \& 40$ ps for $\mathrm{D}>1$.

b) Flop setup time $=23 \mathrm{ps}$.

c) NOR gate delay $=25 \mathrm{ps}$

** Simulation with Post-layout extracted netlist, $1 \mathrm{~V}$ supply at $75^{\circ} \mathrm{C}$.

be similarly slowed down to speed up the sum output.

Additional overheads exist in the integrator that further contribute to the overall delay. Firstly, integrators are often required to be reset at start-up in order to drive them to a known state. Hence, a NOR gate is required at all outputs of the pipeline in order to synchronously reset the integrator. This increases the load on sum outputs of all the adders and introduces a skew between the sum and sum outputs of $\mathrm{A}_{0}$ $\mathrm{A}_{\mathrm{N}-1}$. A second overhead is a higher load of two flip-flops on $S_{1}$ outputs (see Fig. 3). This can be understood from Figs. 1 and 2 where it can be seen that the integrator output is fed back and also needs to be sent to the next MASH stage as $\Delta \Sigma$ modulators rarely employ a first order shaping function. Replicating the flops results in a lower delay than having one flop to drive both the feedback path and the next MASH stage.

With this understanding of the factors that affect the overall delay, the integrator pipeline of Fig. 3 was simulated using combinational static CMOS logic and the pipeline depth was varied from one to five and the worst case delay was calculated in each case. The 1-bit adders were implemented as carry select adders while the flip-flops used were standard transmission gate flip-flops (TGFF). The simulations were carried out in a standard $65 \mathrm{~nm}$ CMOS technology at $1 \mathrm{~V}$ supply and $75^{\circ} \mathrm{C}$ using low- $\mathrm{V}_{\mathrm{T}}$ general purpose devices. The simulations use post-layout extracted netlists for the flops and adders. Table I shows the obtained delays and overall effective throughput as a function of pipeline depth.

Table I shows that although an effective speed greater than $>10 \mathrm{GHz}$ can be achieved with a 1-bit pipeline, the number of flops required to just pipeline the inputs of a first order 12bit to 3-bit MASH modulator (Fig. 2) is $\sim 140$ which makes $5 \mathrm{GHz}$ clock distribution very challenging. The average 1-b adder delays flattens to an optimal 39 ps per 1-b adder for pipeline depth $>3$ and results in speeds of up to $6.9 \mathrm{GHz}$ with an optimal flop count. Between these two ends of the solution space, a pipeline depth of two and three can be used for speeds between 7.4-9.1 GHz with a moderate increase in number of flip-flops $(40 \%)$ and a $12 \%$ less optimal average 1-b adder delay. It can also be noted that the fixed overhead delays that result from the flop delay/setup time and the reset NOR gate account for at least $30 \%$ of the overall path delay. 


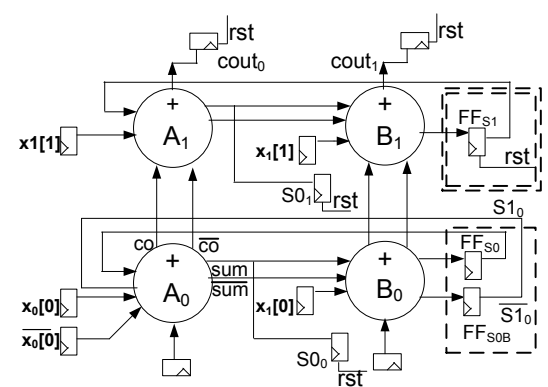

Fig. 4. A 2-bit pipeline with optimization. Single dashed box shows complementary inputs. Double box shows reset moved to the flop.

TABLE II

DELAY COMPARISON WITH ALTERNATIVE LOGIC STYLE FOR 2-BIT PIPELINES.

\begin{tabular}{|c|c|c|c|c|}
\hline $\begin{array}{c}\text { FF } \\
\text { Type }\end{array}$ & $\begin{array}{l}\text { Logic } \\
\text { Style }\end{array}$ & $\begin{array}{c}\text { Delay } \\
\text { (ps) }\end{array}$ & $\begin{array}{c}\text { Eff. } \\
\text { Speed } \\
(\mathbf{G H z})\end{array}$ & Impact \\
\hline TGFF & $\begin{array}{c}\text { Combinational } \\
\text { CMOS }\end{array}$ & 219 & 9.1 & $\begin{array}{l}- \text { Baseline } \\
\text { measurement. }\end{array}$ \\
\hline $\begin{array}{l}\text { TGFF } \\
\text { with } \\
\text { Reset }\end{array}$ & $\begin{array}{l}\text { Combinational } \\
\text { CMOS \& } \\
\text { complementary } \\
\text { inputs }\end{array}$ & 204 & 9.8 & - None. \\
\hline TGFF & $\begin{array}{c}\text { Ratioed } \\
\text { (pseudo-NMOS) }\end{array}$ & 198 & 10.1 & $\begin{array}{l}-80 \% \text { increase } \\
\text { in power. } \\
- \text { Reduced noise } \\
\text { margin. }\end{array}$ \\
\hline TSPCR & $\begin{array}{l}\text { Combinational } \\
\text { CMOS \& } \\
\text { complementary } \\
\text { inputs } \\
\end{array}$ & 201 & 9.9 & $\begin{array}{c}\text { - Dynamic nodes } \\
\text { in TSPCRFF. }\end{array}$ \\
\hline $\begin{array}{c}\text { Tristated } \\
\text { Inverter } \\
\text { Latch }\end{array}$ & $\begin{array}{l}\text { Pre-charged } \\
\text { Domino }\end{array}$ & 195 & 10.2 & $\begin{array}{l}\text { - Increased design } \\
\text { complexity. } \\
\text { - Doubled clock } \\
\text { load. } \\
\text { - Reduced noise } \\
\text { margin. }\end{array}$ \\
\hline $\begin{array}{l}\text { TGFF } \\
\text { with } \\
\text { Reset }\end{array}$ & $\begin{array}{c}\text { Complementary } \\
\text { Pass Trans. } \\
\text { with keepers }\end{array}$ & 213 & 9.4 & - None. \\
\hline
\end{tabular}

\section{Delay Optimization and Alternative Logic STYLES}

Since static CMOS logic is extremely robust with superior noise immunity, the simulation results presented in the previous section serve as baseline measurement against which further delay optimization or alternative logic styles can be compared. This section presents some alternatives for delay improvement and discusses the trade-offs involved. In this section, a 2-bit pipeline depth as shown in Fig. 4 is used as a reference for all discussions.

Firstly, there exists a possibility to replicate $\mathrm{FF}_{\mathrm{S} 0}$ of Fig. 3 such that both the complementary inputs can be directly provided to adder $\mathrm{A}_{0}$ in Fig. 4 (single dashed square). This gives back one inverter delay of $12 \mathrm{ps}$, however, the fanout for the sum output of $\mathrm{B}_{0}$ is now higher compared to Fig. 3, which reduces this gain to about 4 ps. Thus, providing complementary inputs to the first adder is only moderately

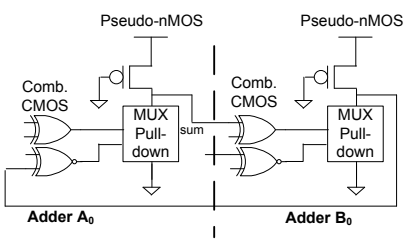

(a)

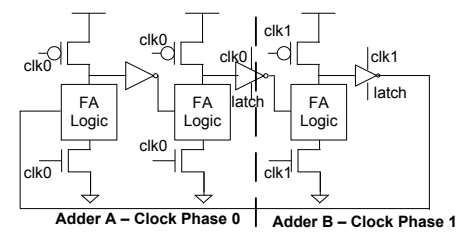

(b)
Fig. 5. (a) Pseudo-nMOS implementation. (b) Pre-charged domino implementation.

beneficial due to the feedback nature of the critical path.

A second optimization strategy involves moving the reset logic inside the flop, so as to make it an asynchronous reset instead (Fig. 4, double dashed square). This gives back 25 ps from the NOR gate (Table I) but increases the flop delay by 15 ps (7 ps in setup time, 8 ps in flop output delay). This results in an overall 10-ps delay improvement and combined with the foregoing optimization of providing complementary inputs yields an improvement of $14 \mathrm{ps,} \mathrm{with} \mathrm{only} \mathrm{a} \mathrm{marginal}$ increase in flop area.

Static TGFFs are not the fastest flops, and instead, using a True Single Phase Clocked Register (TSPCR) FF offers the benefits of a reduced setup time and a single clock phase. TSPCRFF shows a 10 ps improvement for the same clock load as that of a TGFF and coupled with the complementary style of providing the inverted inputs to $\mathrm{A}_{0}$, an overall 17 ps improvement is achieved. However, TSPCRFF being a dynamic flop suffers from lower noise margins.

Alternatively, every alternate CMOS gate in the path can be replaced with a ratioed (pseudo-NMOS) logic to reduce the adder load as shown in Fig. 5(a). The full swing is restored after every alternate gate. This yields a 20 ps overall improvement but at a cost of $80 \%$ higher power resulting from the static current in this logic style.

Lastly, a pre-charged domino logic based style was also simulated using a two clock phase system as shown in Fig. 5(b). Adder A operations are performed in the first clock phase while adder B works on the second. Since the number of adders in the critical path is $\mathrm{D}+1$ for a pipeline depth $\mathrm{D}$, having a 2-pipeline depth (odd number of additions) is inefficient as the additions cannot be equally spread out over both the clock phases. Nevertheless, this kind of an implementation results in an average 1-bit adder delay of $40 \mathrm{ps}$ and overall path delay of $195 \mathrm{ps}$ in a 2-bit pipeline. The 23 ps improvement achieved comes at a cost of increased circuit complexity and additional overheads related to the two-phase clocking.

Table II shows the relative comparison of delays between all these discussed styles. The table demonstrates that pure combinational static logic with complementary inputs ready and a TGFF with an asynchronous reset yields a frequency very close to $10 \mathrm{GHz}$. In order to achieve speeds above $10 \mathrm{GHz}$, reduced swing logic or dynamic logic is required, which comes with a high power penalty and/or an impact on noise immunity. Moreover, the speed improvement in these logic styles is less than 5\% compared to the best case delay 


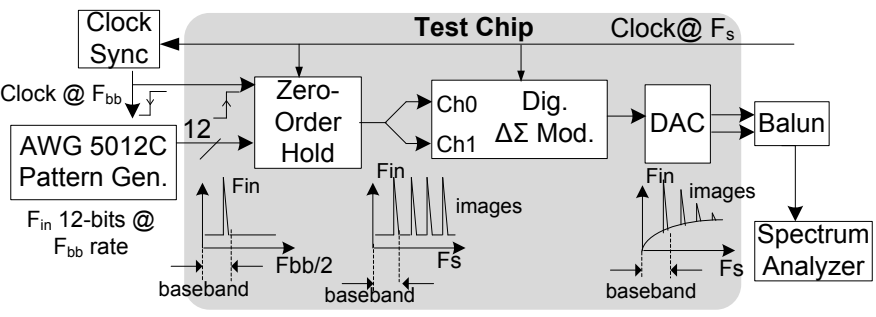

Fig. 6. Measurement setup for the DDSM working at $2 \mathrm{~F}_{\mathrm{s}}$ with the expected spectrum at the output of every block.

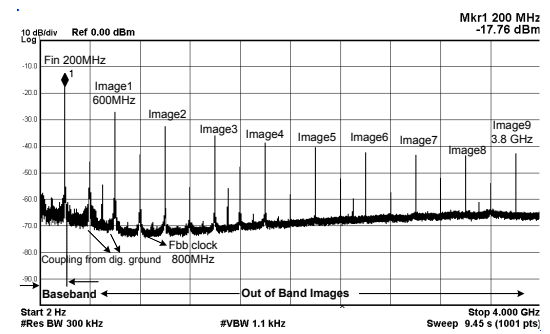

Fig. 7. Measured $8 \mathrm{GHz}$ operation with $\mathrm{F}_{\mathrm{s}}=4 \mathrm{GHz}, \mathrm{F}_{\mathrm{bb}}=800 \mathrm{MHz}$ and input tone, $F_{\text {in }}=200 \mathrm{MHz}$. The fundamental tone and its 9 images are seen.

obtained using static logic. Hence, these styles would be less preferred for implementing very high speed two-channel interleaved $\Delta \Sigma$ modulators.

\section{Measurement Results}

A prototype of a second order 2-channel interleaved MASH 12-bit input 3-bit output modulator was implemented in standard $65 \mathrm{~nm}$ CMOS using combinational static logic and using a 2-bit pipeline of Fig. 3. A DAC was also integrated in this chip. Fig. 6 shows the measurement setup used. A 12-bit input tone of frequency $F_{i n}$ is sent in to the chip at a rate $F_{b b}$ using a pattern generator. Internal to the chip, this data is upsampled to a $\mathrm{F}_{\mathrm{s}}$ rate by a zero-order hold operation and then directly fed to both the the modulator channels, in effect shorting the two channels. To simplify the testing, the $\left(2 \mathrm{~F}_{\mathrm{s}} / \mathrm{F}_{\mathrm{bb}}\right)-1$ images generated from the upsampling and the shorting are not filtered since this is sufficient to verify the modulator operation. Fig. 7 shows the $8 \mathrm{GHz}$ operation of the modulator $(1 \mathrm{~V}$ supply) and the DAC. The DAC dynamic performance at $8 \mathrm{GHz}$ has been presented in [5]. Beyond $8 \mathrm{GHz}$, the DAC dynamic performance deteriorates but nevertheless this still allows the checking of modulator operation. Fig. 8 shows that the modulator continues to operate at $9 \mathrm{GHz}, 1 \mathrm{~V}$ supply with the input signal and its images observed as expected. Table III shows that this modulator achieves the highest speed as compared to the existing works in literature.

\section{CONCLUSION}

This paper has presented the critical path analysis of a two-channel interleaved MASH $\Delta \Sigma$ modulator and the delay bottlenecks in the modulator were identified. Simulations show that static CMOS combinational logic with transmission gate flip-flops is an optimum choice of logic style when aiming for a "multi-GHz" operation. With measured speed of MASH $\Delta \Sigma$

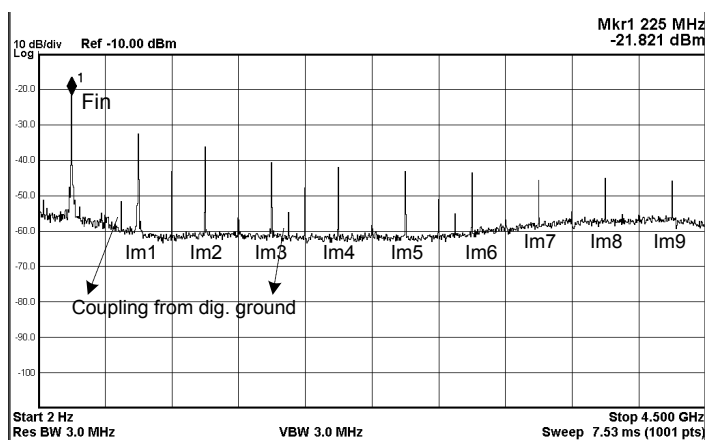

Fig. 8. Measured $9 \mathrm{GHz}$ operation with $\mathrm{F}_{\mathrm{bb}}=900 \mathrm{MHz}, \mathrm{F}_{\text {in }}=225 \mathrm{MHz}$ and its 9 images.

TABLE III

COMPARISON WITH $\Delta \Sigma$ MODULATORS HAVING $>2.5$-GHZ SPEED.

\begin{tabular}{|l|c|c|c|c|c|}
\hline Paper & {$[1]$} & {$[2]$} & {$[3]$} & {$[4]$} & $\begin{array}{c}\text { This } \\
\text { Work }\end{array}$ \\
\hline $\begin{array}{l}\text { Modulator } \\
\text { Type }\end{array}$ & $\begin{array}{c}\text { Error } \\
\text { Feedback }\end{array}$ & MASH & $\begin{array}{c}\text { Error } \\
\text { Feedback }\end{array}$ & MASH & $\begin{array}{c}\text { Interleaved } \\
\text { MASH }\end{array}$ \\
\hline Technology & $90 \mathrm{~nm}$ & $65 \mathrm{~nm}$ & $90 \mathrm{~nm}$ & $0.13 \mu \mathrm{m}$ & $65 \mathrm{~nm}$ \\
\hline Input/Output Bits & $10 / 3$ & $5 / 3$ & $13 / 2$ & $12 / 3$ & $12 / 3$ \\
\hline Order & 2 & 3 & 3 & 2 & 2 \\
\hline Speed (GHz) & 3.6 & 5.4 & 4 & 2.6 & 9 \\
\hline Power (mW) & 11 & $>50$ & 15 & 40 & 68 \\
\hline
\end{tabular}

modulators reaching $9 \mathrm{GHz}$ in $65 \mathrm{~nm}$ CMOS, speed exceeding $10 \mathrm{GHz}$ can be achieved in further scaled CMOS technologies for use in wideband wireless communication.

\section{REFERENCES}

[1] P. Seddighrad, A. Ravi, M. Sajadieh, H. Lakdawala and K. Soumyanath, "A $3.6 \mathrm{GHz} 16 \mathrm{~mW} \Delta \Sigma$ DAC for a $802.11 \mathrm{n} / 802.16 \mathrm{e}$ transmitter with $30 \mathrm{~dB}$ digital power control in 90nm CMOS“, Proc. IEEE European SolidState Circuits Conf., 2008, pp. 202-205.

[2] A. Pozsgay, T. Zounes, R. Hossain, M. Boulemnakher, V. Knopnik and S. Grange, "A Fully Digital 65nm CMOS Transmitter for the 2.4-to-2.7GHz WiFi/WiMAX Bands using $5.4 \mathrm{GHz} \Delta \Sigma$ RF DACs", IEEE. Int. Solid-State Circ. Conf. Dig. Tech. Papers, 2008, pp. 360-619.

[3] A. Frappe, A. Flament, B. Stefanelli, A. Kaiser and A. Cathelin, "An All-Digital RF Signal Generator Using High-Speed $\Delta \Sigma$ Modulators" , IEEE J. Solid State Circuits, vol. 44, pp. 2722-2732, Oct. 2009.

[4] A. Jerng and C. Sodini, "A Wideband $\Delta \Sigma$ DigitalRF Modulator for High Data Rate Transmitters", IEEE J. Solid State Circuits, vol. 42, pp. 1710-1722, Aug. 2007.

[5] A. Bhide, O. E. Najari, B. Mesgarzadeh and A. Alvandpour, "An 8-GS/s 200-MHz Bandwidth 68-mW $\Delta \Sigma$ DAC in 65-nm CMOS", IEEE. Trans. Circuits SystemsII: Express Briefs, vol. 60, pp. 387-391, July. 2013.

[6] P. Madoglio et al., "A 2.5-GHz, 6.9-mW, 45-nm-LP CMOS, $\Delta \Sigma$ Modulator Based on Standard Cell Design With Time-Interleaving“, IEEE J. Solid State Circuits, vol. 45, pp. 1410-1420, July 2010. 